\title{
PROPRIEDADES FÍSICAS DO SORVETE DE BAUNILHA ELABORADO COM SUBSTITUTOS DE GORDURA E SACAROSE
}

\author{
ELIZABETH HARUMI NABESHIMA * \\ EVELYN DE SOUZA OLIVEIRA ** \\ JORGE MINORU HASHIMOTO * \\ MARISA N. H. JACKIX ***
}

\begin{abstract}
Sorvetes de baunilha preparados com substitutos de gordura (3-5\%) e de sacarose (6-10\%) foram comparados, mediante análises físicas e calóricas, com sorvete convencional. A combinação de $4 \%$ de "Simplesse" e $8 \%$ de "Litesse" evidenciaram efeito sinergístico para retardar a velocidade de fusão. Menores valores de densidade aparente e maior redução calórica foram obtidos com concentrações próximas a 3 e 6\% (p/p) de "Simplesse" e "Litesse", respectivamente. Baixas concentrações de "Litesse" $(6 \%, \mathrm{p} / \mathrm{p})$ e altas de "Simplesse" (5\%, p/p) resultaram em maior tempo de início de fusão. A utilização desses substitutos permitiu a obtenção de produtos com baixos teores calórico e de gordura e características físicas semelhantes às de sorvete convencional.
\end{abstract}

PALAVRAS-CHAVE: SORVETES; SUBSTITUTOS DE GORDURA; SACAROSE; SIMPLESSE; LITESSE.

\section{INTRODUÇÃO}

A popularidade das sobremesas geladas deve-se ao fato de tratar-se de produto pronto para consumo, amplamente disponível e de valor nutritivo, que apresenta formas, cores e sabores atrativos $(1,9)$. No entanto, a aceitabilidade de determinado produto não depende somente do sabor e da aparência, mas de suas complexas propriedades físicas que afetam as respostas sensoriais, como a maciez, mastigabilidade, cremosidade e velocidade de fusão (12).

* Doutorandos, Bolsistas CNPq, Departamento de Tecnologia de Alimentos (DTA), Faculdade de Engenharia de Alimentos (FEA), Universidade Estadual de Campinas (UNICAMP), Campinas, SP.

** Professor Adjunto, Departamento de Tecnologia de Alimentos, Faculdade de Ciências Farmacêuticas, Universidade de Minas Gerais, Belo Horizonte, MG.

*** Professor Adjunto, DTA, FEA, UNICAMP, Campinas, SP.

(e-mail: ehnabeshima@hotmail.com). 
Alimentos para fins especiais obtidos pela redução ou ausência de açúcar ou ainda pela redução ou substituição de gorduras ("low-fat", "light", "lite", etc) encontram mercado promissor, atendendo pessoas sensíveis aos apelos de marketing ou preocupadas com melhores condições de saúde, redução de peso ou a manutenção de aparência atraente. $O$ incentivo proporcionado pela demanda destes produtos tem permitido 0 aprimoramento da tecnologia e o desenvolvimento de melhores ingredientes e métodos de produção (5).

Os consumidores estão mais conscientes da importância da alimentação para a saúde, e com isso alterando hábitos alimentares, mas de modo que não traga sacrifício da satisfação psicológica. O aumento da popularidade de alimentos com teor de gordura reduzido provocou maior demanda de produtos no segmento da indústria de sorvetes (8).

Para atender a expectativa de consumidores que procuram nos produtos com baixa caloria a mesma qualidade dos produtos convencionais, como sabor e textura adequados, ausência de colesterol e baixo teor de gorduras, instituições públicas e privadas têm dedicado crescentes esforços para atingir estes requisitos.

O objetivo deste trabalho foi avaliar o efeito de substituto de gordura ("Simplesse" 100) e de substituto de sacarose ("Litesse") nas propriedades físicas de sorvete de baunilha, bem como compará-las com as de sorvete convencional contendo gordura e açúcar.

\section{MATERIAL E MÉTODOS}

Foram utilizados como matéria-prima para produção dos sorvetes de baunilha, leite em pó desnatado (Molico - Indústrias Nestlé), gordura vegetal hidrogenada ("Primor", ponto de fusão $=42{ }^{\circ} \mathrm{C}$, Santista Alimentos), maltodextrina (Mor-rex 1910-10\% de glucose DE; Sorbitol líquido $70 \%$, porcentagem máxima de açúcares redutores $=0,06$ e viscosidade a $40^{\circ} \mathrm{C}$ de $100 \mathrm{cP}$, Indústria GETEC), acessulfame k (Indústrias Hoechst $A G)$, aspartame (Indústrias Holland Sweetener Company), mistura de emulsificantes e estabilizantes (Cremodan ${ }^{\mathrm{TM}}$ SIM-B, Indústrias Danisco Ingredients Brasil Ltda), saborizante de baunilha (Indústrias Sun Foods), corante amarelo de tartrazina, polímero de dextrose ("Litesse", viscosidade de $300 \mathrm{cP}$ a $40^{\circ} \mathrm{C}$, Indústrias PFIZER), concentrado protéico de soro de leite ("Simplesse" 100, Indústrias Nutra Sweet Kelco Company) e sacarose (açúcar refinado União). 
Os componentes dos sorvetes elaborados (Quadro 1) distinguiram-se do sorvete controle ou padrão pela substituição total da sacarose por edulcorantes (mistura de 0,015 p/p aspartame e 0,015 p/p acessulfamek) e substituto de sacarose, "Litesse" (6, 8 ou 10\% da formulação). A redução da gordura de $5 \mathrm{p} / \mathrm{p}$ para $2 \mathrm{p} / \mathrm{p}$ foi compensada pela adição de substituto de gordura, "Simplesse" 100 (3, 4 ou 5\% da formulação). Os produtos "Litesse" e "Simplesse" 100 foram adicionados em porcentagens definidas em ensaios preliminares e com base nas recomendações do fabricante.

\section{QUADRO 1 - COMPOSIÇÃO DO SORVETE PADRÃO (CONTROLE) E DOS SORVETES FORMULADOS COM SUBSTITUTO DE SACAROSE (LITESSE) E DE GORDURA (SIMPLESSE)}

\begin{tabular}{|c|c|c|}
\hline Ingredientes & $\begin{array}{c}\text { Sorvete padra } 0 \\
(p / p)\end{array}$ & $\begin{array}{l}\text { Sorvetes formulados } \\
(\mathrm{p} / \mathrm{p})\end{array}$ \\
\hline Leite em $p$ desnatado & 12 & 12 \\
\hline Gordura vegetal hidrogenada & 5 & 2 \\
\hline Sacarose & 8 & - \\
\hline Maltodextrina & 5 & 5 \\
\hline Sorbitol & 2 & 2 \\
\hline Edulcorantes Aspartame & - & 0,015 \\
\hline Acessulfame-k & - & 0,015 \\
\hline Emulsificante/estabilizante & 0,8 & 0,8 \\
\hline Aroma & 0,1 & 0,1 \\
\hline Corante & 0,075 & 0,075 \\
\hline Litesse $^{a}$ & - & $6-10$ \\
\hline Simplesse $^{b}$ & - & $3-5$ \\
\hline
\end{tabular}

a A concentração de "Litesse" utilizada foi de 6, 8 e 10\%, conforme recomendação do fabricante.

b A concentração de "Simplesse" 100 utilizada foi de 3, 4 e 5\%, conforme recomendação do fabricante.

O Fluxograma 1 apresenta as etapas do processamento do sorvete, sendo calculada a quantidade de cada ingrediente de modo a se obter 2 quilos 
de mistura. Todos os ingredientes, exceto o emulsificante, o aromatizante, o corante e a gordura foram misturados num recipiente. Em seguida, adicionou-se água destilada, procedeu-se à homogeneização e adicionouse o emulsificante.

\section{FLUXOGRAMA 1 - PROCESSO DE FABRICAÇÃO DE SORVETE}

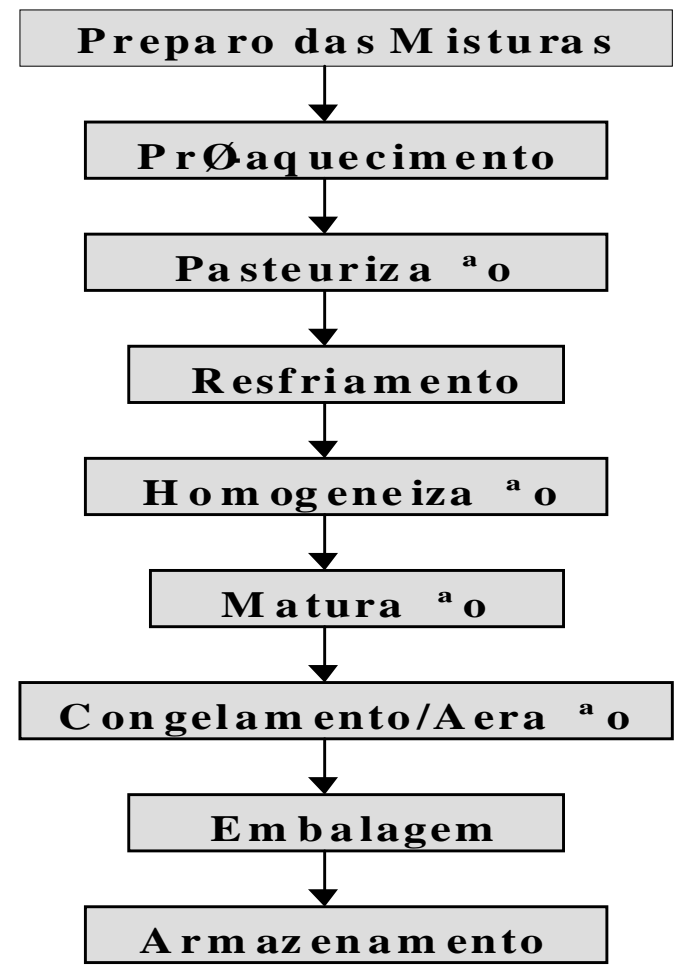

A mistura foi agitada manualmente e aquecida lentamente até $60^{\circ} \mathrm{C}$, quando adicionou-se a gordura previamente fundida $\left(60^{\circ} \mathrm{C}\right)$. A pasteurização foi efetuada no próprio recipiente de mistura a $82^{\circ} \mathrm{C} / 2 \mathrm{~min}$. Imediatamente após, a mistura foi resfriada até $70^{\circ} \mathrm{C}$ com o auxílio de banho de gelo e homogeneizada em homogeneizador K (Janke \& Kunkel, lea-Wirk) por 2 minutos. Depois de resfriá-la até $25^{\circ} \mathrm{C}$ foram adicionados o saborizante e o corante procedendo-se nova homogeneização por mais 2 minutos. 
As misturas foram colocadas em geladeira a $7^{\circ} \mathrm{C}$, durante 4 horas, para sofrer o processo de maturação. Em seguida, as mesmas foram congeladas/aeradas, utilizando-se sorveteira (Inadal, capacidade de 6 litros) do Laboratório de Laticínios do Departamento de Tecnologia de Alimentos/ UNICAMP, com tempo de batimento de 40 minutos. Os sorvetes obtidos foram colocados em embalagens plásticas, de formato retangular e armazenados em freezer horizontal a $4^{\circ} \mathrm{C}$.

Durante o preparo dos sorvetes analisou-se o $\mathrm{pH}$ das misturas, de acordo com o método adotado pelo Instituto Adolfo Lutz (6). Após o preparo dos sorvetes foi analisada a densidade aparente, que é a medida do ar incorporado ao sorvete após batimento, expressa em gramas/litro (3).

As propriedades de fusão foram avaliadas de acordo com o método de LEE \& WHITE (7). Amostras de sorvete de $70 \mathrm{~g}$ foram colocadas em peneira de material plástico, com funil e proveta graduada acoplados. A temperatura ambiente foi mantida a $25 \pm 1{ }^{\circ} \mathrm{C}$ e o volume de sorvete drenado medido em intervalos de 10 minutos. Foram construídos gráficos do tempo versus volume de sorvete drenado. A velocidade de drenagem (inclinação) e o tempo inicial de drenagem (intersecção com o eixo dos $\mathrm{x}$ ) foram obtidos utilizando-se regressão linear.

A redução do valor calórico foi calculada em função da porcentagem de cada ingrediente na formulação e com base na Resolução - RDC n.40, de 22 mar. 2001 do Ministério da Saúde (4), que especifica os seguintes valores para carboidratos (sacarose, maltodextrina, exceto polióis) $4,0 \mathrm{Kcal} / \mathrm{g}$; polióis 2,4 Kcal/g; polidextrose $1,0 \mathrm{Kcal} / \mathrm{g}$; proteínas 4,0 Kcal/g e lipídios 9,0 Kcal/g. O valor calórico do "Simplesse" (2 Kcal/g) foi calculado conforme apresentado na especificação do produto (NutraSweet Company) e citado por CÂNDIDO \& CAMPOS (5).

Para estudar o efeito combinado das variáveis independentes nas respostas utilizou-se o delineamento fatorial completo $2^{2}$, contendo duas variáveis e dois níveis com 7 ensaios (4 combinações e ponto central em triplicata), conforme BARROS NETO et al. (2). As variáveis independentes estudadas foram "Litesse" e "Simplesse" 100 e as variáveis dependentes densidade aparente, redução calórica e velocidade de fusão (Quadro 2). Efetuou-se a análise de variância (ANOVA) dos dados, utilizando o programa STATISTICA (11) e as médias dos resultados das análises físicas foram comparadas pelo teste de Tukey. 


\section{QUADRO 2 - DEFINIÇÕES E NÍVEIS DAS VARIÁVEIS INDEPENDENTES DO PLANEJAMENTO FATORIAL $2^{2}$ COM PONTOS CENTRAIS}

\begin{tabular}{|c|c|c|c|c|}
\hline \multirow{2}{*}{ Ensaio } & \multicolumn{2}{|c|}{ Valores Codificados } & \multicolumn{2}{c|}{ Valores Reais } \\
\cline { 2 - 5 } & $\mathbf{X}_{\mathbf{1}}$ & $\mathbf{X}_{\mathbf{2}}$ & $\begin{array}{c}\text { Litesse } \\
(\mathbf{p} / \mathbf{p})\end{array}$ & $\begin{array}{c}\text { Simplesse } \\
(\mathbf{p} / \mathbf{p})\end{array}$ \\
\hline 1 & - & - & 6 & 3 \\
2 & + & - & 10 & 3 \\
3 & - & + & 6 & 5 \\
4 & + & + & 10 & 5 \\
5 & 0 & 0 & 8 & 4 \\
6 & 0 & 0 & 8 & 4 \\
7 & 0 & 0 & 8 & 4 \\
\hline
\end{tabular}

\section{RESULTADOS E DISCUSSÃO}

3.1 COMPARAÇÃO DOS RESULTADOS DAS ANÁLISES FÍSICAS E DA REDUÇÃO CALÓRICA DAS DIFERENTES FORMULAÇÕES DE SORVETES EM RELAÇÃO À AMOSTRA PADRÃO

$\mathrm{O}$ pH das misturas variou de 6,47 a 6,56 (Tabela 1) mostrando-se de acordo com os valores encontrados na literatura (10).

\section{TABELA 1 - DETERMINAÇÕES FÍSICAS E REDUÇÃO CALÓRICA DAS AMOSTRAS DE SORVETES}

\begin{tabular}{|c|c|c|c|c|c|c|c|c|}
\hline AnA画ses & Controle & 1 & 2 & 3 & 4 & 5 & 6 & 7 \\
\hline $\mathrm{pH}$ & 6,50 & 6,56 & 6,50 & 6,47 & 6,50 & 6,50 & 6,56 & 6,55 \\
\hline Densidade aparente $(\mathrm{g} / \mathrm{L})$ & 880,6 & 681,9 & 785,4 & 783,0 & 776,6 & 736,4 & 747,3 & 770,9 \\
\hline In cio fus ${ }^{\underline{a}} \circ$ (min) & 29,3 & 28,6 & 24,1 & 30,4 & 26,0 & 27,6 & 31,2 & 29,2 \\
\hline Veloc.fus ${ }^{\underline{a}} \circ(\mathrm{mL} / \mathrm{min})$ & 0,70 & 1,43 & 1,29 & 1,26 & 1,25 & 1,27 & 1,00 & 0,99 \\
\hline S lidos solœeis totais & 33,8 & 30,8 & 34,8 & 32,8 & 36,8 & 33,8 & 33,8 & 33,8 \\
\hline Calorias (Kcal/g) & 149,8 & 102,8 & 106,8 & 106,8 & 110,8 & 106,8 & 106,8 & 106,8 \\
\hline Redu ${ }^{a}$ o cal rica (\%) & 0,0 & 31,4 & 28,7 & 28,7 & 26,0 & 28,7 & 28,7 & 28,7 \\
\hline
\end{tabular}

Verificou-se que os valores de densidade aparente para todos os tratamentos (Tabela 1) foram menores do que o valor encontrado para o controle. O menor valor, ou seja, o sorvete mais aerado correspondeu ao ensaio 1 (681,9 g/L). Os sorvetes obtidos neste estudo enquadram-se nos limites da legislação vigente que exige densidade aparente mínima de $475 \mathrm{~g} / \mathrm{L}$ para o produto (3). 
Em todos os ensaios houve redução calórica de $26,0 \%$ a 31,4\% em relação à fórmula padrão e redução de gordura de $60 \%$ (Tabela 1). Por demonstrar redução calórica maior que $25 \%$, o produto pode ser classificado como alimento para fins especiais, modificado com "valor energético reduzido" e por evidenciar redução de gordura superior a $30 \%$, o produto também pode ser classificado como sendo de "baixo teor de gordura".

As amostras de sorvetes foram submetidas ao teste de fusão a fim de se determinar o efeito dos tratamentos nas propriedades de fusão. Conforme pode ser visualizado na Figura 1 e na Tabela 1, a velocidade de derretimento observada para a amostra padrão (controle) foi menor em relação aos demais ensaios, mostrando-se bastante similar entre os diferentes ensaios.

\section{FIGURA 1 - VARIAÇÃO DO VOLUME DE SORVETE DRENADO, OBTIDO NAS DIFERENTES FORMULAÇÕES, EM TEMPERATURA AMBIENTE $\left(25^{\circ} \mathrm{C}\right)$}

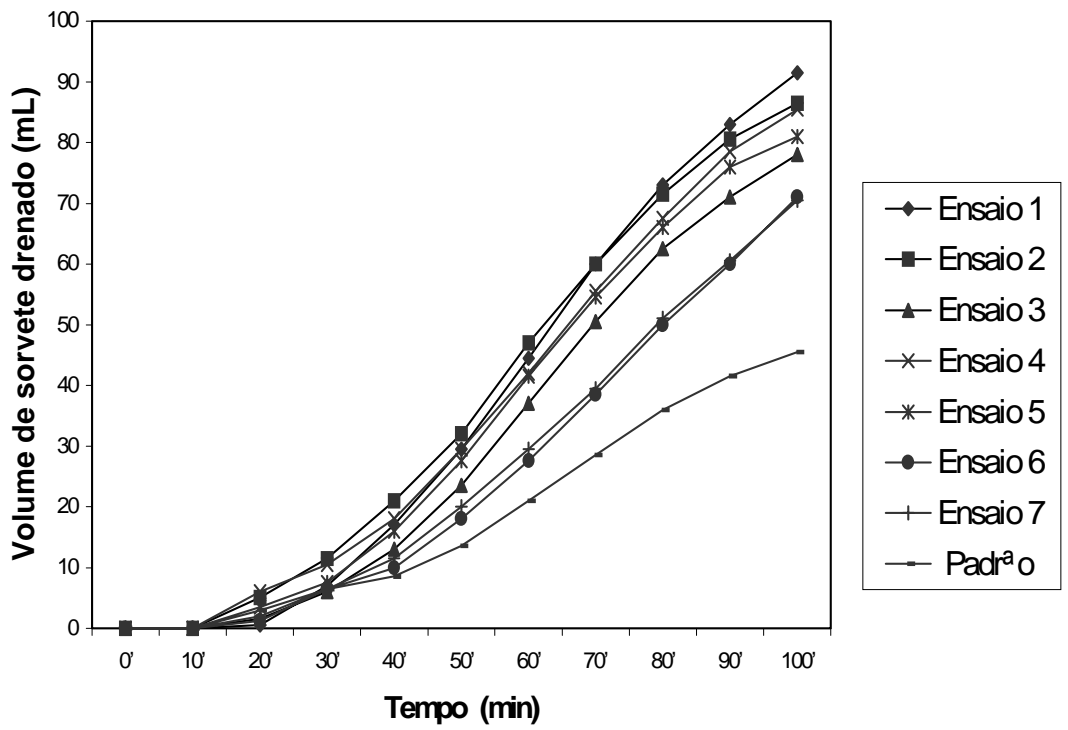


Teores mais baixos de "Simplesse" (ensaios 1 e 2) parecem acelerar a velocidade de derretimento, enquanto que teores maiores (ensaios 3 e 4) tendem a retardar o derretimento. A combinação de 4\% de "Simplesse" e $8 \%$ de "Litesse" (ensaios 6 e 7, principalmente) apresentaram efeito sinergístico para retardar o derretimento. Com relação ao tempo de início de fusão todas as formulações apresentaram valores similares ao controle. Os dados, previamente selecionados, forneceram mediante regressão linear $\left(R^{2}>0,99\right)$ a velocidade de drenagem (inclinação da reta) e o tempo inicial de drenagem (intersecção com o eixo dos $\mathrm{x}$ ).

\subsection{ANÁLISE DO PLANEJAMENTO FATORIAL COMPLETO $2^{2}$ COM PONTOS CENTRAIS}

A Tabela 2 apresenta os dados de densidade aparente e início de fusão das diferentes formulações de sorvete de baunilha.

\section{TABELA 2 - RESULTADOS DO PLANEJAMENTO FATORIAL $2^{2}$ COM PONTOS CENTRAIS}

\begin{tabular}{|c|c|c|c|c|}
\hline \multirow[b]{2}{*}{ Ensaio } & \multicolumn{2}{|c|}{ Vari/Æeis Independentes } & \multicolumn{2}{|c|}{ VariÆteis Dependentes } \\
\hline & $\begin{array}{l}\text { Litesse } \\
(p / p)\end{array}$ & $\begin{array}{c}\text { Simplesse } \\
(p / p)\end{array}$ & $\begin{array}{c}\text { Densidade } \\
\text { Aparente } \\
(\mathrm{g} / \mathrm{L})\end{array}$ & $\begin{array}{l}\text { In cio Fus }{ }^{\mathrm{a}} \mathrm{o} \\
\text { (minutos) }\end{array}$ \\
\hline 1 & 6 & 3 & 681,9 & 28,6 \\
\hline 2 & 10 & 3 & 785,4 & 24,1 \\
\hline 3 & 6 & 5 & 783,0 & 30,4 \\
\hline 4 & 10 & 5 & 776,6 & 26,0 \\
\hline 5 & 8 & 4 & 736,4 & 27,6 \\
\hline 6 & 8 & 4 & 747,3 & 31,2 \\
\hline 7 & 8 & 4 & 770,9 & 29,2 \\
\hline
\end{tabular}

Os resultados, com relação à densidade aparente, aplicados à regressão multivariada evidenciaram equação linear capaz de explicar $91,8 \%$ da variação observada em torno da média. A equação linear permite inferir que a densidade aparente foi influenciada principalmente pela interação entre as duas variáveis ("Litesse" e "Simplesse") que apresentou efeito negativo sobre esta propriedade:

$$
Y=754,50+24,27 X_{1}+23,07 X_{2}-27,47 X_{1} X_{2}
$$


De acordo com a Tabela 3 pode-se inferir que a equação linear foi estatisticamente adequada.

\section{TABELA 3 - ANÁLISE DE VARIÂNCIA PARA O AJUSTE DO MODELO LINEAR}

\begin{tabular}{|c|c|c|c|c|c|}
\hline $\begin{array}{l}\text { Fonte de } \\
\text { Varia } 0\end{array}$ & 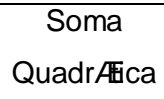 & $\mathrm{GL}$ & $\begin{array}{c}\text { MØdia } \\
\text { Quadr/Fica }\end{array}$ & $\begin{array}{c}\mathrm{F} \\
\text { calculado }\end{array}$ & $\mathrm{Fc} / \mathrm{Ft}$ \\
\hline Regress ${ }^{\underline{a}} 0$ & 7506,43 & 3 & 2502,14 & $11,23^{*}$ & 2,08 \\
\hline Res duo & 668,21 & 3 & 222,74 & & \\
\hline Falta Ajuste & 46,21 & 1 & 46,21 & & \\
\hline Erro Puro & 622,01 & 2 & 311,00 & & \\
\hline Total & 8174,64 & 6 & 23,80 & & \\
\hline
\end{tabular}

* Significativo ao nível de $90 \%$.

$\mathrm{Ftab}_{0,90,3,3}=5,39 ; \mathrm{R}^{2}=0,918 ; \mathrm{GL}=$ Grau de Liberdade; $\mathrm{Fc}=\mathrm{F}$ calculado; $\mathrm{Ft}=\mathrm{F}$ tabelado.

Na Figura 2 são apresentados os gráficos de superfície de resposta e de contorno para a densidade aparente. Analisando os gráficos verifica-se que a faixa para a obtenção de sorvetes com menor densidade aparente, ou com maior aeração, situa-se na região que apresenta menores valores de "Litesse" $(p / p)$ e de "Simplesse" ( $p / p)$. Maiores valores desta propriedade foram obtidos na região de menor teor de "Simplesse" e maior teor de "Litesse" ou, maior teor de "Simplesse" e menor teor de "Litesse".

Com relação à variável início de fusão, o modelo linear não foi estatisticamente significativo, pois apresentou coeficiente de correlação de 0,63 , ou seja, o modelo explicou apenas a variação de $63 \%$ da resposta. Sendo assim, considerou-se conveniente utilizá-la apenas para estabelecer a tendência de variação da resposta em função das variáveis independentes na região estudada e para delimitar nova área de experimentação, mas não para predizer valores exatos do conteúdo de início de fusão.

De acordo com a Figura 3 verifica-se que os maiores valores de tempo para o início da fusão foram obtidos nas regiões com maiores valores de "Simplesse" e menores valores de "Litesse". O maior tempo decorrido para o início da fusão, verificado experimentalmente, foi de 31,2 minutos, nas concentrações médias de "Litesse" ( 8 p/p) e de "Simplesse" (4 p/p). 


\section{FIGURA 2 - EFEITO DA ADIÇÃO DE "LITESSE" E "SIMPLESSE" SOBRE A DENSIDADE APARENTE}
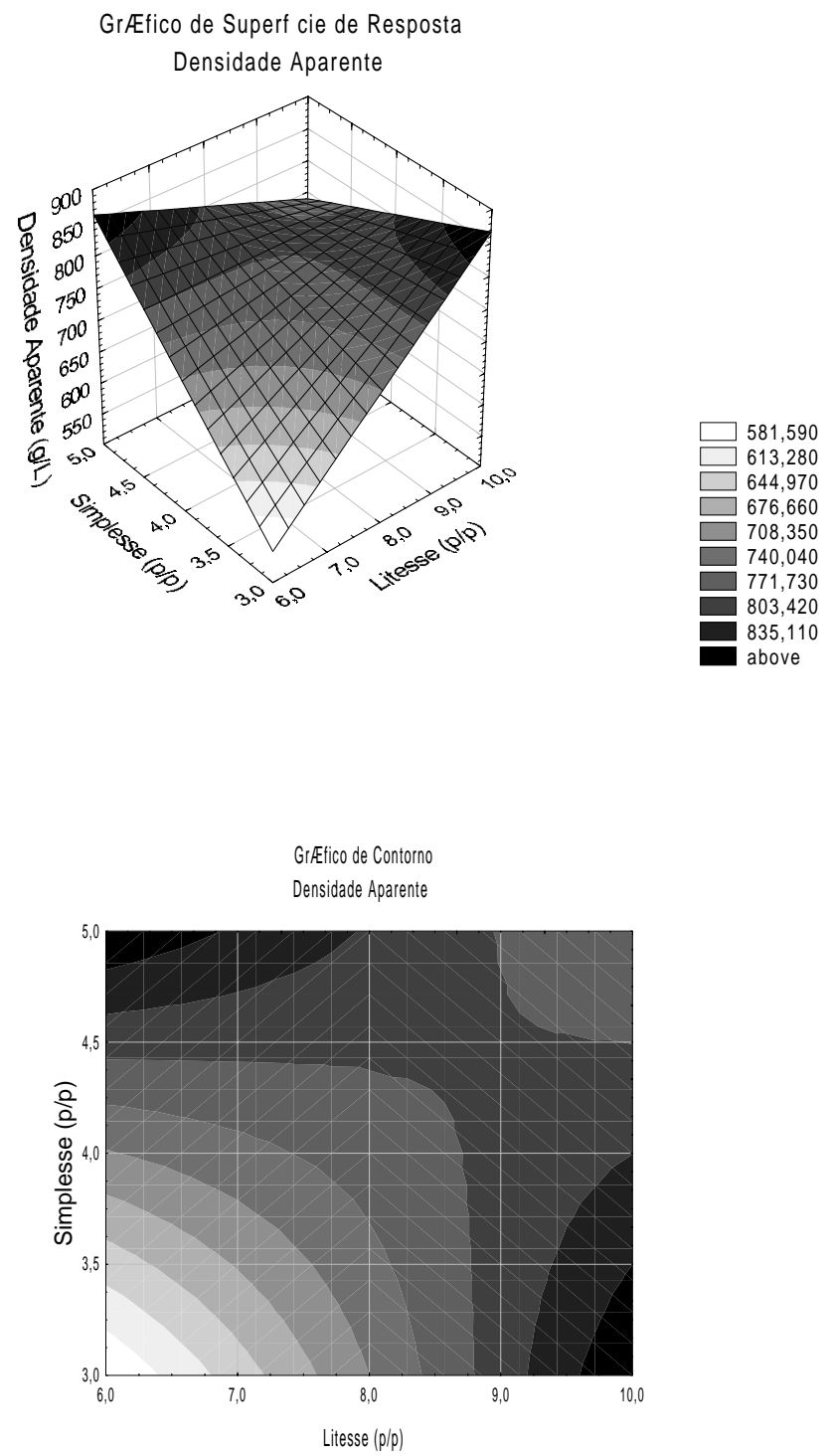


\section{FIGURA 3 - EFEITO DA ADIÇÃO DE "LITESSE" E "SIMPLESSE" SOBRE O INÍCIO DE FUSÃO}
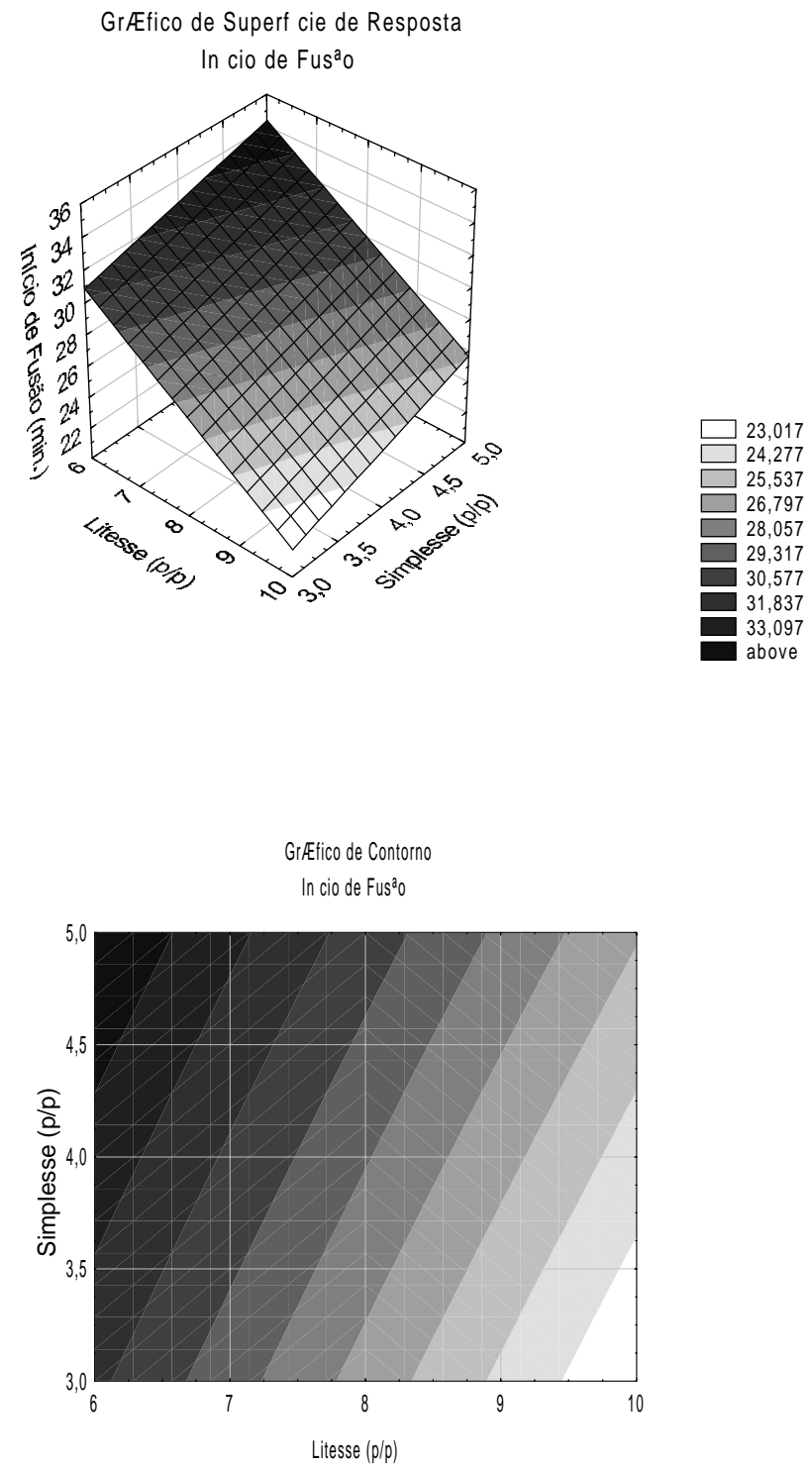


\section{CONCLUSÃO}

Considerando as faixas de "Simplesse" e "Litesse" estudadas pode-se concluir que para se obter sorvete com menor densidade aparente devese trabalhar com concentrações próximas a 3,0 e 6,0\% de "Simplesse" e "Litesse", respectivamente.

Os valores de densidade aparente mostraram-se menores para todas as formulações em relação à amostra padrão (controle).

Menores valores de "Simplesse" promoveram maior velocidade de derretimento, enquanto que teores maiores apresentaram tendência a retardar o derretimento.

A velocidade de derretimento dos sorvetes formulados com substitutos de gordura e sacarose foi maior que a obtida com a amostra padrão.

A utilização do substituto de gordura "Simplesse" e do substituto de sacarose "Litesse" nas formulações de sorvete estudadas ocasionaram redução de gordura de $60 \%$ e calórica de $26,0 \%$ a $31,4 \%$ em relação à formulação padrão.

O modelo linear não foi significativo para a variável início de fusão sugerindose que seja testado o modelo quadrático para esta variável.

É possível produzir sorvete com baixo teor calórico e de gordura, com características físicas semelhantes às de sorvete convencional, utilizando "Simplesse" e "Litesse".

\section{Abstract \\ PHYSICAL PROPERTIES OF VANILLA ICE CREAM PREPARED WITH FAT AND SUCROSE SUBSTITUTES}

Prepared vanilla ice creams with fat (3-5\%) and sucrose (6-10\%) substitute were compared through physical and caloric analyses to conventional ice cream. The combination of $4 \%$ of Simplesse and $8 \%$ of Litesse showed sinergistic effect to delay the melting speed. Lower overrun and higher caloric reduction values were obtained with concentrations at 3 and $6 \%(p / p)$ of Simplesse and Litesse, respectively. Low concentrations of Litesse $(6 \%, \mathrm{p} / \mathrm{p})$ and high of Simplesse $(5 \%, \mathrm{p} / \mathrm{p})$ resulted in higher melting start time. The use of these substitutes allowed to obtain products with low calorie degree and of fat with physics characteristics similar to the standard ice cream.

KEY-WORDS: ICE CREAMS; FAT SUBSTITUTES; SUCROSE; SIMPLESSE LITESSE. 


\section{REFERÊNCIAS}

1 ARBUCKLE, W.S. Ice cream. $3^{\text {rd }}$ ed. Westport: Avi., 1977. 517p.

2 BARROS NETO, B.; SCARMÍNIO, I.S.; BRUNS, R.E. Planejamento e otimização de experimentos. Campinas: Ed. Unicamp, 1995.

3 BRASIL. Ministério da Saúde. Secretaria de Vigilância Sanitária. Portaria n. 379, de 26 de abril de 1999. Regulamento técnico para fixação de identidade e qualidade de gelados comestíveis, preparados, pós para o preparo e bases para gelados comestíveis. Diário Oficial [da] República Federativa do Brasil, Brasília, 29 de abril de 1999.

4 BRASIL. Ministério da Saúde. Secretaria de Vigilância Sanitária. Resolução RDC n. 40, de 21 de março de 2001. Regulamento técnico para rotulagem nutricional obrigatória de alimentos e bebidas embalados. Diário Oficial [da] República Federativa do Brasil, Brasília, 22 de março de 2001.

5 CÂNDIDO, L.M.B.; CAMPOS, A.M. Alimentos para fins especiais: dietéticos. São Paulo: Varela, 1995. p. 423.

6 INSTITUTO ADOLFO LUTZ. Normas analíticas do Instituto Adolfo Lutz: métodos químicos e físicos para análise. 2. ed. São Paulo, 1976. v. 1.

7 LEE, F.Y.; WHITE, C.H. Effect of ultrafiltration retentates and whey protein concentrates on ice cream quality during storage. Journal of Dairy Science, Champaign, v.74, p.1170, 1991.

8 KANTOR, M. A. Light dairy products: the need and the consequences. Food Technology, Chicago, v.44, p.81, 1990.

9 MORETTI, M.H. Elaboração de sorvetes. Campinas: Fundação Tropical de Pesquisas e Tecnologia, 1977. 121 p.

10 SCHMIDT, K.; LUNDY, A.; REYNOLDS, J.; YEE, L.N. Carbohydrate or protein based fat mimicker effects on ice milk properties. Journal of Food Science, Chicago, v.58, n.4, p.761-799, 1993.

11 STATSOFT. Statistica for windows: release 5.0 A. USA, 1995. 
12 THOMAS, E.L. Structure and properties of ice cream emulsions. Food Technology, Chicago, v.1, p.41-48, 1981. 